\title{
Image Denoising using Neighbors Variation with Wavelet
}

\author{
S D Ruikar \\ Research Scholar \\ SGGS IET \\ Nanded, India
}

\author{
D D Doye \\ Professor E\&TC \\ SGGS IET \\ 2nd line of address
}

\begin{abstract}
The image gets corrupted by Additive White Gaussian Noise during the process of acquisition, transmission, storage and retrieval. Denoising refers to suppressing the noise while retaining the edges and other important detailed structures as much as possible. This paper presents a general structure of the recovery of images using a combination of variation methods and wavelet analysis. The variation formulation of the problem allows us to build the properties of the recovered signal directly into the analytical machinery. The efficient wavelet representation allows us to capture and preserve sharp features in the signal while it evolves in accordance with the variation laws. We propose the three different variation model for removing noise as Horizontal, vertical and Cluster. Horizontal and Vertical variation model obtained the threshold at each decomposed level of Wavelet. Cluster variation model moving mask in different wavelet sub band. This proposed scheme has better PSNR as compared to other existing technique.
\end{abstract}

\section{General Terms}

Image Processing

\section{Keywords}

Horizontal Variation, Vertical Variation, Cluster Variation, Wavelet, Noise, etc.

\section{INTRODUCTION}

Image processing is a field that continues to grow, with new applications being developed at an ever increasing pace; it includes digital cameras, intelligent traffic monitoring, handwriting recognition on checks, signature validation and so on. It is a fascinating and exciting area to be involved in today with application areas ranging from the entertainment industry to the space program. One of the most interesting aspects of this information revolution is the ability to send and receive complex data that transcends ordinary written text. Visual information, transmitted in the form of digital images, has become a major method of communication for the $21^{\text {st }}$ century. During transmission and acquisition, images are often corrupted by various noises. The aim of image denoising is to reduce the noise level while keeping the image features as much as possible [1] [2]. The image denoising approaches can put into two broad categories like spatial domain and frequency domain [3]. In the spatial domain approach the pixels of an image are manipulated directly, such as median filter, averaging filter, point processing, Weiner filter, etc [4]. The frequency domain approach is based on modifying the transformed image such as Fourier transform and Wavelet transform of an image. One of the widely used techniques is the wavelet thresholding. This scheme performs on noisy images as small coefficients in the high frequencies. A thresholding can be done by setting these small coefficients to zero; will eliminate much of the noise in the image [5] [6].
The denoising scheme using proposed variation model is shown in figure (1).

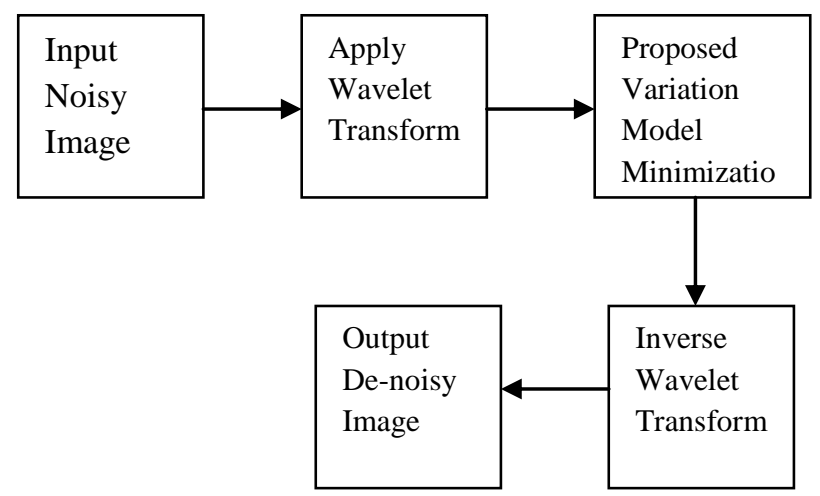

Fig 1: Variation Model with wavelet

\section{RELATED WORK ON TOTAL VARIATION IMAGE DENOISING}

Image denoising is an important research field in image processing. It is often considered as a pre-processing step for other image tasks such as image segmentation, image registration and so on. Image restoration includes many aspects, for example denoising, deblurring, in painting and colorization etc. In the last two decades many authors have introduced certain tools for the image denoising problem. Introduction in a classic paper by Rudin, Osher, and Fatemi, total variation minimizing models have become one of the most popular and successful methodology for image restoration [7]. Total Variation is a well known image prior introduced by Rudin, Osher and Fatemi (ROF). For a differential function $f: \Omega=[0,1]^{2} \rightarrow R$ it is computed as $\|f\|_{T V}=\int_{\Omega}|\nabla f|$, and can be extended to the space $B V\left([0,1]^{2}\right)$ that contains functions with discontinuities. The total variation is used as a regularization to denoise an image by solving the strictly convex problem

$$
\min _{f \in B V\left([0,1]^{2}\right)} \frac{1}{2}\left\|f-f_{0}\right\|^{2}+\lambda\|f\|_{T V}
$$

as originally proposed by ROF. The regularization weight $\lambda$ should be tuned to match the noise level contaminating f0. Several algorithms have been proposed to solve this problem. Such primal, dual, or primal-dual schemes for denoising are often a building block for solving more complex inverse problems. A. Haddad [8] begins with a review of well-known 
properties of $B V$. They define the space of functions of bounded variation $B V$. This space is endowed with an isotropic norm $\|\| B$.$V . They fix the dimension to 2$ and choose $\Omega=R^{2}$ so that concentrates on dilatation. $\mathrm{S}$ Osher[9] propose a new model for image restoration and image decomposition into cartoon and texture, based on the total variation minimization of Rudin, Osher, and Fatemi, and on oscillatory functions, which follows results of Meyer[10], involving $\mathrm{H}^{-1}$ norm. This model performs better on textured images, and the "residual" component has less structure than in the standard TV model. Fang fang Dong [11] proposed vectorial algorithm to some applications besides image denoising. They stated that vectorial algorithm can be used in many problems which need the $l 1$ regularization. Yang Wang and Haomin Zhou [12] propose a denoising algorithm for medical images based on a combination of the total variation minimization scheme and the wavelet scheme. This method offers effective noise removal in real noisy medical images while maintaining sharpness of objects. More importantly, this scheme allows us to implement an effective automatic stopping time criterion. Another improvement is the multiscale fitting parameters targeting denoising in the high frequency domain, which yields a significant reduction in number of iterations needed to achieve the desired denoising as well as a small performance improvement in terms of PSNR on simulated noisy images. Kossi Edoh and John Paul Roop[13] , presents an adaptive multilevel total variation (TV) method for image denoising which utilizes TV partial differential equation (PDE) model and exploits the multiresolution properties of wavelets. They develop a fast method which combines TV denoising with denoising from wavelet compression, which is known to produce results which are superior to either method alone. Gabriele Steidl, Joachim Weickert, Thomas Brox, Pavel Mrazek, And Martin Welk [14] investigate under which conditions one can prove equivalence between four discontinuity preserving denoising techniques in the 1-D case: soft wavelet thresholding, TV diffusion, TV regularization, and SIDEs. Starting from a simple two-pixel case they were able to derive analytical solutions. Antonin Chambolle [15] proposes an algorithm for minimizing the total variation of an image, and provides a proof of convergence. He has work on applications to image denoising, zooming, and the computation of the mean curvature motion of interfaces. Paul Rodriguez, Brendt Wohlberg [16] proposes a simple but flexible method for solving the generalized vector-valued TV (VTV) functional, which includes both the $l^{2}$-VTV and $l^{1}$-VTV regularizations as special cases, to address the problems of deconvolution and denoising of vector-valued images with Gaussian or salt-and pepper noise. Yilun Wang, Junfeng Yang, Wotao Yin, And Yin Zhang [17], proposes, analyze and test an alternating minimization algorithm for recovering images from blurry and noisy observations with total variation (TV) regularization. Their algorithm arises from a new halfquadratic model applicable to not only the anisotropic but also isotropic forms of total variation discretizations. Banazier A.
Abrahim, Yasser Kadah[18], proposes a new speckle reduction method and coherence enhancement of ultrasound images based on method that combines total variation (TV) method and wavelet shrinkage. In this method, a noisy image is decomposed into sub bands of LL, LH, HL, and $\mathrm{HH}$ in wavelet domain. LL sub band contains the low frequency coefficients along with less noise, which can be easily eliminated using TV based method. More edges and other detailed information like textures are contained in the other three sub bands. They propose a shrinkage method based on the local variance to extract them from high frequency noise.

David C. Dobsony and Curtis R. Vogel [19], analyzes the convergence of an iterative method for solving nonlinear minimization problems. The iterative method involves a lagged diffusivity approach in which sequences of linear diffusion problems are solved. Global convergence in a finitedimensional setting is established, and local convergence properties, including rates and their dependence on various parameters, are examined. Rick Chartrand [20] considers the problem of differentiating a function specified by noisy data. Regularizing the differentiation process avoids the noise amplification of finite-difference methods. He used total variation regularization, which allows for discontinuous solutions. The resulting simple algorithm accurately differentiates noisy functions, including those which have a discontinuous derivative.

\section{DISCRETE WAVELET TRANSFORM}

Wavelets are the functions generated from one single function by dilations and translations [21] [22] where dilation means scaling the wavelet and translation meaning shifting the wavelet. The wavelet expansion set is not unique. A wavelet system is a set of building blocks to construct or represent a signal or function. It is a two- dimensional expansion set, usually a basis for some class one or higher dimensional signals.

The wavelet can be represented by a weighted sum of shifted scaling function $\varphi(2 t)$ as,

$$
\psi(t)=\sum_{n} h_{1}(n) \sqrt{2} \varphi(2 t-n), \quad \mathrm{n} \in \mathrm{Z} \text {---- (1) }
$$

For some set of coefficient $h_{1}(n)$, this function gives the prototype or mother wavelet $\psi(t)$ for a class of expansion function of the form

$$
\psi_{j, k}(t)=2^{j / 2} \psi\left(2^{j} t-k\right)
$$

Where $2^{j}$ the scaling of is $t, 2^{-j}$ is the translation in $t$, and $2^{j / 2}$ maintains the $L^{2}$ norms of the wavelet at different scales. The construction of wavelet using a set of scaling function $\varphi_{k(t)}$ and $\psi_{j, k}(t)$ that

could span all of $L^{2}(R)$ therefore function $g(t) \in L^{2}(R)$ can be written as

$$
g(t)=\sum_{k=-\infty}^{\infty} c(k) \varphi_{k}(t)+\sum_{j=0}^{\infty} d(j, k) \psi_{j, k}(t) \text {------ (3) }
$$

First summation in the above equation gives a function that is low resolution of $g(t)$, for each increasing index $\mathrm{j}$ in the 
second summation, a higher resolution function is added which gives increasing details. The function

$d(j, k)$ indicates the differences between the translation index $k$, and the scale parameter $j$. Figure ( 2 a) shows the structure of two stages down sampling filter banks in terms of coefficients.

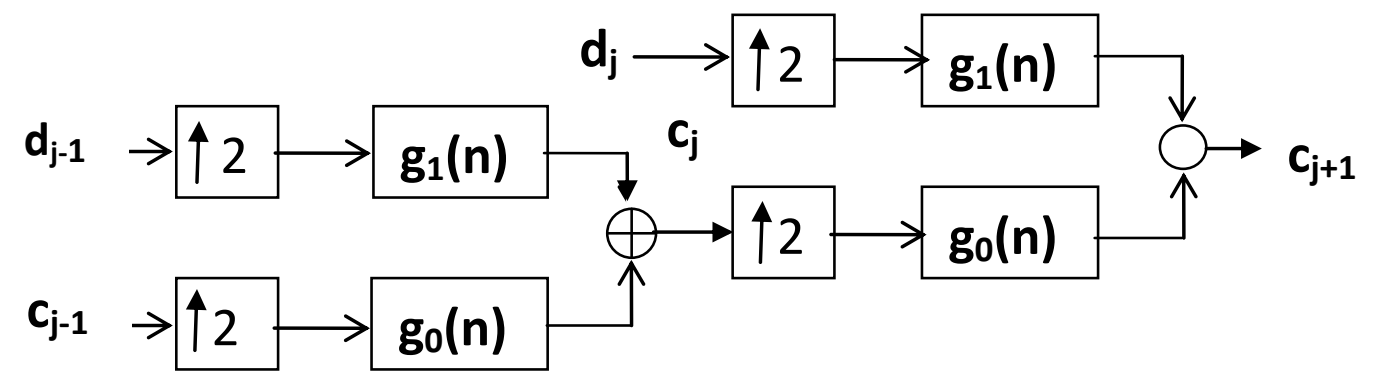

(a) Two stages down sampling Filter bank

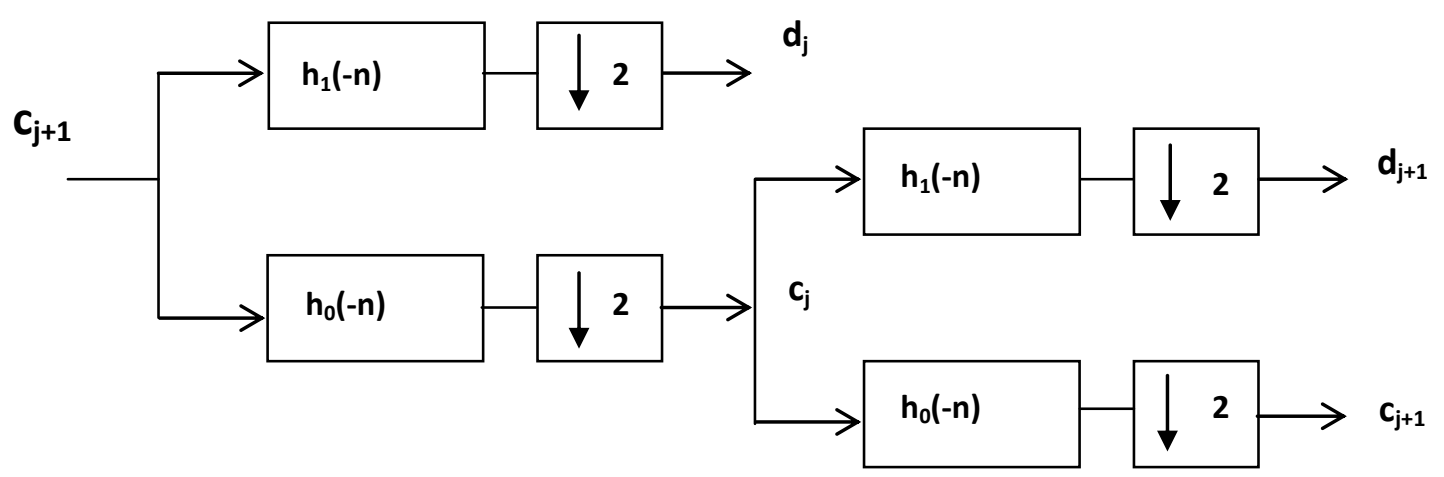

(b) Two stages up sampling filter.

Fig 2: down sampling and up sampling filter.

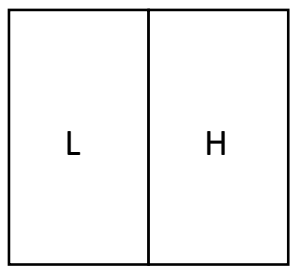

\begin{tabular}{|c|c|}
\hline LL & $\mathrm{HL}$ \\
\hline $\mathrm{LH}$ & $\mathrm{HH}$ \\
\hline
\end{tabular}

\begin{tabular}{|l|l|l|}
\hline LL & LH & \multirow{2}{*}{ HL } \\
\cline { 1 - 2 } LH & HH & \\
\hline \multicolumn{2}{|c|}{ LH } & HH \\
\hline
\end{tabular}

(c)Two dimensional decomposition Fig 3: Two-dimensional wavelet transform
A reconstruction of the original fine scale coefficient of the signal made from a combination of the scaling function and wavelet coefficient at a course resolution is derived by considering a signal in the $\mathrm{j}+1$ scaling function space $f(t) \in v_{j+1}$. Figure (2 b) shows the structure of two stages up sampling filter banks in terms of coefficients i.e. synthesis from coarse scale to fine scale [23] [24] [25].

The DWT is identical with a hierarchical sub band system where the sub bands are logarithmically spaced in frequency and represent octave-band decomposition. By applying DWT, the image is actually divided i.e. decomposed into four sub bands and critically sub-sampled as shown in Figure (3 a). These four sub bands arise from separable applications of vertical and horizontal analysis filters for wavelet decomposition as shown in Figure ( $3 \mathrm{~b})$. The filters h0 and h1 shown in Figure (2) are one-dimensional Low Pass Filter (LPF) and High Pass Filter (HPF), respectively. Thus, decomposition provides sub bands corresponding to different resolution levels and orientation.

These sub bands labeled LH, LH, HL and HH represent the finest scale wavelet coefficients, i.e. detail images while the sub band LL corresponds to coarse level coefficients, i.e. approximation image. To obtain the next coarse level of wavelet coefficients, the sub band LL alone is further decomposed and critically sampled using similar filter bank shown in Figure (2). This results in two-level wavelet decomposition as shown in Figure $(3 \mathrm{c})$. The decomposed image can be reconstructed using a reconstruction (i.e. Inverse DWT) or synthesis filter.

\section{PROPOSED VARIATION TECHNIQUE}

Image denoising is one of the measure issues in image processing, not only because it plays a key preliminary role in many computer vision systems, but also because it is probably the simplest way to address the fundamental issue of image 
modeling, as a starting point towards more complex tasks like deblurring, demosaicking, in painting, etc. We propose the new method to determine variation around the neighbor and modify the pixel according to the energy in that block. A discretized gradient for an image $f \in R^{N}$ is defined as $\nabla f(i, j)=\left(\partial_{x} f(i, j), \partial_{y} f(i, j) \quad\right.$ where

$\partial_{x} f(i, j)=\left\{\begin{array}{lr}f(i+1, j)-f(i, j) & \text { if } 0 \leq \mathrm{i}<\mathrm{n}-1 \\ 0 & \text { otherwise }\end{array}\right.$

$\partial_{y} f(i, j)=\left\{\begin{array}{lr}f(i, j+1)-f(i, j) & \text { if } 0 \leq \mathrm{j}<\mathrm{n}-1 \\ 0 & \text { otherwise }\end{array}\right.$

These equations give the gradient with respect to horizontal and vertical axis in image. Figure (4) shows a technique of horizontal, vertical and neighboring variation proposed model where, $\mathrm{X}$ is marked as the location of the pixel in an image.<smiles>[Y4][Y10]CC[Y1]</smiles>

(a)Horizontal Variation

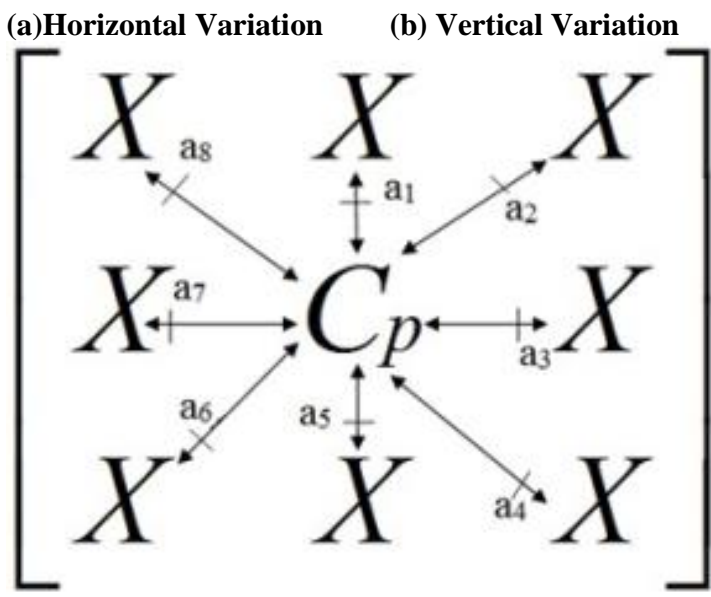

(c) Cluster Variation

Fig 4: Variation Model

\subsection{Horizontal Variation:}

We propose a wavelet based Variation denoising scheme. In our scheme, the wavelet coefficients are selected and modified by applying the horizontal and vertical variation model.

The horizontal variation model applied to the image noisy image patch.

$$
H_{\mathrm{var}}=\sum_{j=1}^{n}\left(X_{i, j}-X_{i, j+1}\right)
$$

Where, $\mathrm{H}_{\mathrm{var}}$ is the horizontal variation. To obtain the regularization parameter of the patch we need to calculate energy of the patch.

Energy $=\frac{1}{\mathrm{~m} * \mathrm{n}} \sum_{i, j}^{m, n} X_{i, j}$

$$
\lambda=\frac{\text { Energy }}{H_{\mathrm{var}}}
$$

Where, $\lambda$ is the regularization parameter of the patch. The coefficient can be modified by

$$
X_{i, j}=\sum_{i, j}^{m, n} \frac{X_{i, j}}{\lambda}
$$

The horizontal coefficients are get modified by regularization parameter.

\section{A. Vertical Variation}

The vertical variation model applied to the image noisy image patch.

$V_{\text {var }}=\sum_{i=1}^{n}\left(X_{i, j}-X_{i+1, j}\right)$

here, $H_{v a r}$ is the horizontal variation. To obtain the regularization parameter of the patch we need to calculate energy of the patch.

$$
\begin{aligned}
& \text { Energy }=\frac{1}{m * n} \sum_{i, j}^{m, n} X_{i, j} \\
& \lambda=\frac{\text { Energy }}{V_{\mathrm{var}}}
\end{aligned}
$$

Where, $\lambda$ is the regularization parameter of the patch. The coefficient can be modified by

$$
X_{i, j}=\sum_{i, j}^{m, n} \frac{X_{i, j}}{\lambda}
$$

The vertical coefficients are get modified by regularization parameter.

\subsection{Cluster Variation}

The cluster variation can be obtained by measuring the variation at its neighbor. Consider $3 * 3$ clusters apply to the wavelet as shown in figure (5).

$$
\left[\begin{array}{ccc}
i-1, j-1 & i-1, j & i-1, j+1 \\
i, j-1 & i, j & i, j+1 \\
i+1, j-1 & i+1, j & i+1, j+1
\end{array}\right]
$$

Fig 5: $3 * 3$ cluster

$$
\begin{aligned}
\mathrm{d}(1) & =(\mathrm{x}(\mathrm{i}, \mathrm{j})-\mathrm{x}(\mathrm{i}-1, \mathrm{j}-1)) \\
\mathrm{d}(2) & =(\mathrm{x}(\mathrm{i}, \mathrm{j})-\mathrm{x}(\mathrm{i}-1, \mathrm{j})) \\
\mathrm{d}(3) & =(\mathrm{x}(\mathrm{i}, \mathrm{j})-\mathrm{x}(\mathrm{i}-1, \mathrm{j}+1)) \\
\mathrm{d}(4) & =(\mathrm{x}(\mathrm{i}, \mathrm{j})-\mathrm{x}(\mathrm{i}, \mathrm{j}-1)) \\
\mathrm{d}(5) & =(\mathrm{x}(\mathrm{i}, \mathrm{j})-\mathrm{x}(\mathrm{i}, \mathrm{j}+1)) \\
\mathrm{d}(6) & =(\mathrm{x}(\mathrm{i}, \mathrm{j})-\mathrm{x}(\mathrm{i}+1, \mathrm{j}-1)) \\
\mathrm{d}(7) & =(\mathrm{x}(\mathrm{i}, \mathrm{j})-\mathrm{x}(\mathrm{i}+1, \mathrm{j})) \\
\mathrm{d}(8) & =(\mathrm{x}(\mathrm{i}, \mathrm{j})-\mathrm{x}(\mathrm{i}+1, \mathrm{j}+1)) \\
d=\frac{1}{k} \sum_{k=1}^{8} d_{k} &
\end{aligned}
$$

The obtained cluster variation coefficients $d$ will be replaced by modified coefficients $x(i, j)$. Repeat this procedure to all sub band of Wavelet Transform and modified the Wavelet coefficient. This $3 * 3$ mask will move through the sub band 
and get modified coefficients. This method will not add any blur in the image.

\section{IMPLEMENTATION AND RESULTS}

We demonstrate the Variation scheme for removing noise. The wavelet Variation scheme allows us to modify the wavelet coefficients primarily in the high frequency domain. The proposed variation model obtained through the horizontal and vertical variation technique gives comparative result at all type of noise. The coefficients modified at any level patch by patch in this technique. The horizontal and vertical variation technique gives us the threshold value at each level of the sub band. These threshold coefficients will further modified by taking inverse Wavelet Transform. The noise is removed by taking the inverse wavelet transform of modified coefficients. The cluster variation is performed by modification of each pixel done at the center pixel level at all direction. This $3 * 3$ mask will be moving in the each sub band of decomposed Wavelet. This modified Wavelet coefficients at each decomposition level using cluster variation will further processed. This sub band will further up sampled by inverse Wavelet Transform. We get better result to all types of noise for this variation based regularization method with wavelet as shown in figure (6). Results show that the wavelet based horizontal Variation scheme preserves details like edges of the images as shown in figure (7). Results of Vertical variation with different noise density are shown in figure (8). Results of cluster variation with different noise density in wavelet domain are shown in figure (9). All results are combined in Table (1) shows the different variation technique with the different noise.

\section{CONCLUSION}

In this paper we propose Horizontal, Vertical and cluster variation technique. This variation model has gives better result when the noise density is increases. The image output of variation model, we have compared with the existing method. It shows that the existing methods introduce blur in the edges, but proposed scheme has preserves the edges. It has good output with high noise density. Its output images visually have immune to noise. In the high Gaussian noise density our proposed scheme has better result in comparison with the existing method. In the high Speckle noise density cluster variation has better result than the existing method. In the Salt and Pepper noise density all proposed scheme has better PSNR as compared to the existing method.

\section{ACKNOWLEDGMENTS}

We thank to Dr A V Nandedkar for his support for making this technique.

\section{REFERENCES}

[1] Shan GAI, Peng LIU, Jiafeng LIU, Xianglong TANG, "A New Image Denoising Algorithm via Bivariate Shrinkage Based on Quaternion Wavelet Transform", Journal of Computational Information Systems November 2010,3751-3760.

[2] Murat Belge, Misha E. Kilmer, and Eric L. Miller, Member, IEEE, "Wavelet Domain Image Restoration with Adaptive Edge-Preserving Regularization", IEEE Transactions On Image Processing, VOL. 9, NO. 4, APRIL 2000 597-608.
[3] S. Annadurai, R. Shanmugalakshmi, "Fundamentals of Digital Image Processing”, Pearson Education, 2008.

[4] David L. Donoho. "De-noising by soft-thresholding." IEEE Trans. on Information Theory, Vol 41, No. 3, May 1995.

[5] D. L. Donoho and I.M. Johnstone. Adapting to unknown smoothness via wavelet shrinkage. The Journal of Amer. Statist. Assoc. 1995, 90(432):1200-1224.

[6] F. Luisier, T. Blu, and M. Unser. A new SURE approach to image denoising: Interscale orthonormal wavelet thresholding. IEEE Trans.Image Processing. 2007, 16(3):593-606.

[7] L. Rudin, S. Osher, and E. Fatem, "Nonlinear Total Variation Based Noise Removal Algorithms", Physical D, 60:259-268, 1992.

[8] A. Haddad, Y. Meyer, "An Improvement of Rudin Osher Fatemi Model", Applied and Computational Harmonic Analysis. 22 (2007) 319-334.

[9] Stanley Osher, Andres Sole, and Luminita Vese, "Image Decomposition and Restoration Using Total Variation Minimization and the $\mathrm{H}-1$ Norm", Multiscale Model. Simulation, 2003 Society for Industrial and Applied Mathematics, Vol. 1, No. 3, Pp. 349-370.

[10] Y. Meyer, Oscillating Patterns in Image Processing and Nonlinear Evolution Equations, Univ. Lecture Ser. 22, Ams, Providence, RI, 2002.

[11] Fang fang Dong, Jeanine Yang, Chunxiao Liu, And DeXing Kong, "A Fast Algorithm For Vectorial TV-Based Image Restoration", SIAM International Journal Of Numerical Analysis And Modeling

[12] Yang Wang and Haomin Zhou, "Total Variation Wavelet-Based Medical Image Denoising", International Journal of Biomedical Imaging Volume 2006, Article ID 89095, Pages 1-6

[13] Kossi Edoh and John Paul Roop, "A Fast Wavelet Multilevel Approach to Total Variation Image Denoising”, International Journal of Signal Processing, Image Processing and Pattern Recognition Vol. 2, No.3, September 2009 pp 57-74.

[14] Gabriele Steidl, Joachim Weickert, Thomas Brox, Pavel Mrazek, and Martin Welk, "On the Equivalence of Soft Wavelet Shrinkage, Total Variation Diffusion, Total Variation Regularization, and Sides", SIAM Journal of Numerical. Analysis., 2004 Society For Industrial And Applied Mathematics Vol. 42, No. 2, Pp. 686-713

[15] Antonin Chambolle, “An Algorithm for Total Variation Minimization and Applications", Journal of Mathematical Imaging and Vision 20: 89-97, 2004 Kluwer Academic Publishers. Manufactured in The Netherlands.

[16] Paul Rodriguez, Brendt Wohlberg, "A Generalized Vector-Valued Total Variation Algorithm”, 2009 IEEE, ICIP 2009, Pp1309-1312.

[17] Yilun Wang, Junfeng Yang, Wotao Yin, and Yin Zhang, "A New Alternating Minimization Algorithm for Total Variation Image Reconstruction", Rice University Technical Report TR 07-10.

[18] Banazier A. Abrahim, Yasser Kadah, "Speckle Noise Reduction Method Combining Total Variation and Wavelet Shrinkage for Clinical Ultrasound Imaging", IEEE 2011. 
[19] David C. Dobsony and Curtis R. Vogel, "Convergence of an Iterative Method for Total Variation Denoising", SIAM Journal of Numerical. Anal. C 1997 Society For Industrial And Applied Mathematics Vol. 34, No. 5, Pp. 1779-1791, October 1997

[20] Rick Chartrand, "Numerical Differentiation of Noisy Non smooth Data", International Scholarly Research Network ISRN Applied Mathematics Volume 2011, Article ID 164564, 11 pages

[21] C Sidney Burrus, Ramesh A Gopinath, and Haitao Guo, "Introduction to wavelet and wavelet transforms", Prentice Hall1997.
[22] S. Mallat, A Wavelet Tour of Signal Processing, Academic, New York, second edition, 1999.

[23] R. C. Gonzalez and R. Elwood's, Digital Image Processing. Reading, MA: Addison-Wesley, 1993.

[24] M. Sonka, V. Hlavac, R. Boyle Image Processing, Analysis, And Machine Vision. Pp10-210 \& 646-670

[25] Raghuveer M. Rao, A.S. Bopardikar Wavelet Transforms: Introduction to Theory and Application Published by Addison-Wesley 2001 pp1-126.

[26] Arthur Jr Weeks, Fundamental of Electronic Image Processing PHI 2005.

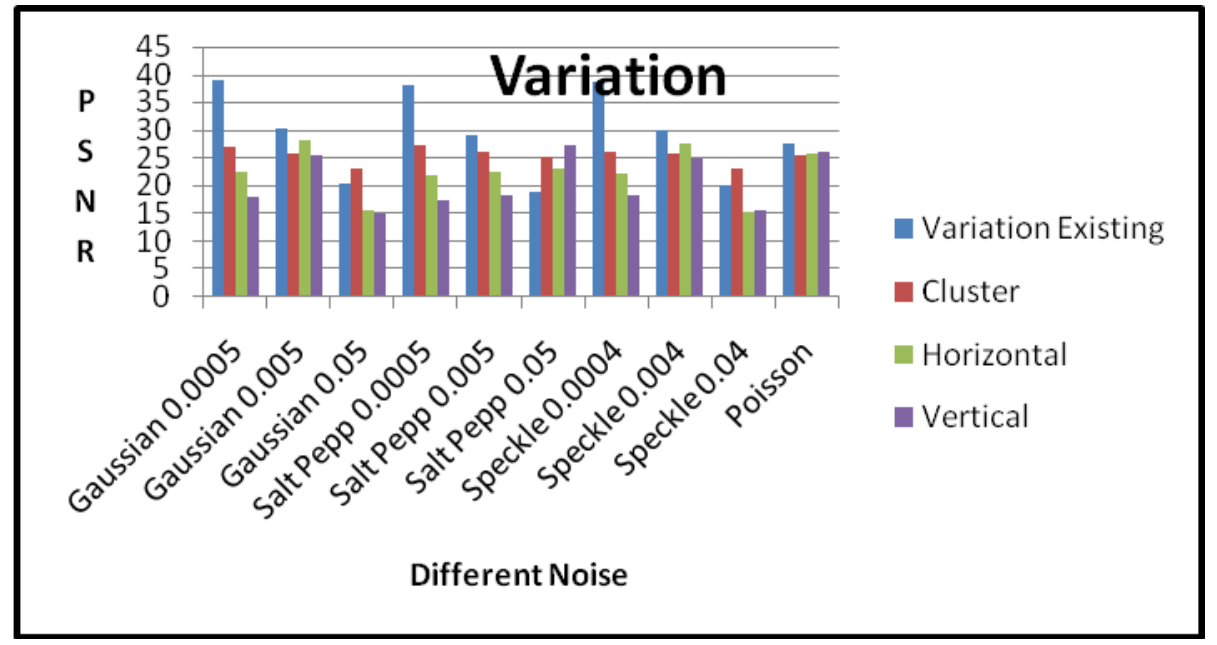

Fig 6: Variation based image denoising with different noise

Table 1: Discrete Variation Model

\begin{tabular}{|c|c|c|c|c|c|c|c|c|c|c|}
\hline \multicolumn{11}{|c|}{ Result for Lena Grayscale image PSNR } \\
\hline \multirow{2}{*}{$\begin{array}{l}\text { Methods / } \\
\text { Different Noise } \\
\text { with different } \\
\text { Variance }\end{array}$} & \multicolumn{3}{|c|}{ Gaussian Noisy Image } & \multicolumn{3}{|c|}{ Salt and Pepper Noisy Image } & \multicolumn{3}{|c|}{ Speckle Noisy Image } & \multirow{2}{*}{$\begin{array}{l}\text { Poisson' } \\
\text { s Noisy }\end{array}$} \\
\hline & $\begin{array}{l}\text { varianc } \\
\text { e } \\
0.0001\end{array}$ & $\begin{array}{l}\text { variance } \\
0.001\end{array}$ & $\begin{array}{l}\text { varianc } \\
\text { e } 0.01\end{array}$ & $\begin{array}{l}\text { noise } \\
\text { density } \\
0.0005\end{array}$ & $\begin{array}{l}\text { noise } \\
\text { density } \\
0.005\end{array}$ & $\begin{array}{l}\text { noise } \\
\text { density } \\
0.05\end{array}$ & $\begin{array}{l}\text { varianc } \\
\text { e } 0.0004\end{array}$ & $\begin{array}{l}\text { varianc } \\
\text { e } 0.004\end{array}$ & $\begin{array}{l}\text { varianc } \\
\text { e } 0.04\end{array}$ & \\
\hline $\begin{array}{l}\text { Variation Existing } \\
\text { Method }\end{array}$ & 39.0432 & 30.3717 & 20.3980 & 38.0274 & 29.0831 & 18.8844 & 38.8144 & 30.0294 & 20.0653 & 27.6249 \\
\hline Cluster Variation & 26.9298 & 25.7157 & 23.1412 & 27.2462 & 26.0150 & 25.0754 & 26.0987 & 25.8306 & 23.1432 & 25.4096 \\
\hline $\begin{array}{l}\text { Horizontal } \\
\text { Variation }\end{array}$ & 22.4632 & 28.2214 & 15.5213 & 21.7780 & 22.5298 & 23.0342 & 22.2532 & 27.6541 & 15.1977 & 25.8700 \\
\hline Vertical Variation & 18.1114 & 25.6088 & 15.0313 & 17.3889 & 18.2763 & 27.2317 & 18.2486 & 24.8501 & 15.6168 & 26.2101 \\
\hline
\end{tabular}
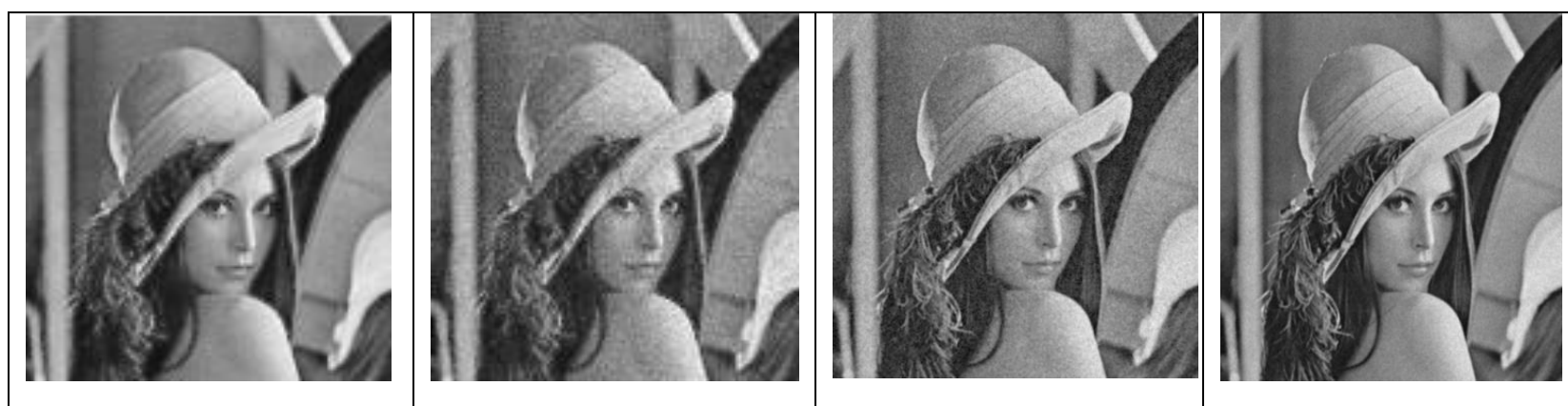


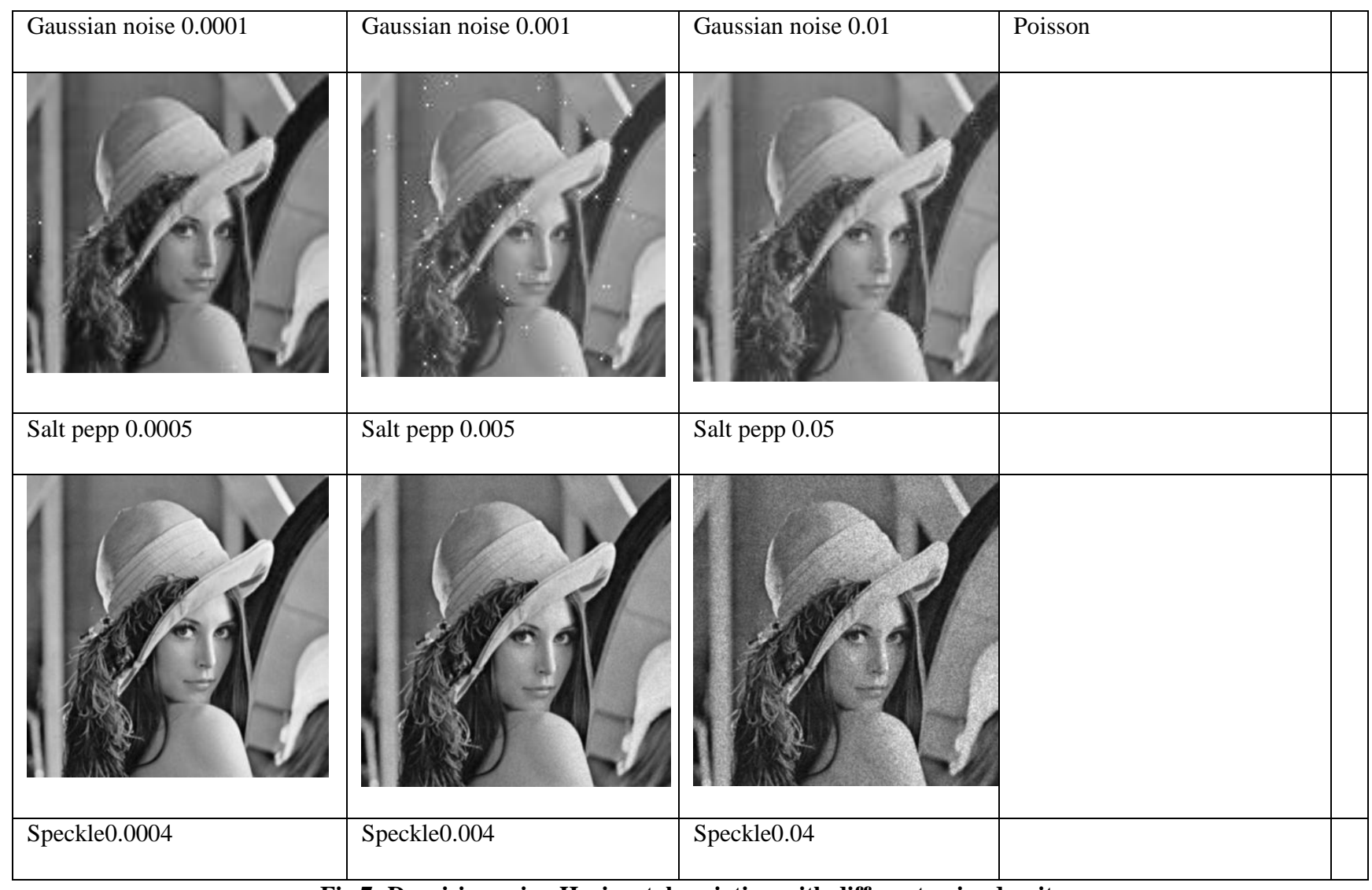

Fig 7: Denoising using Horizontal variation with different noise density

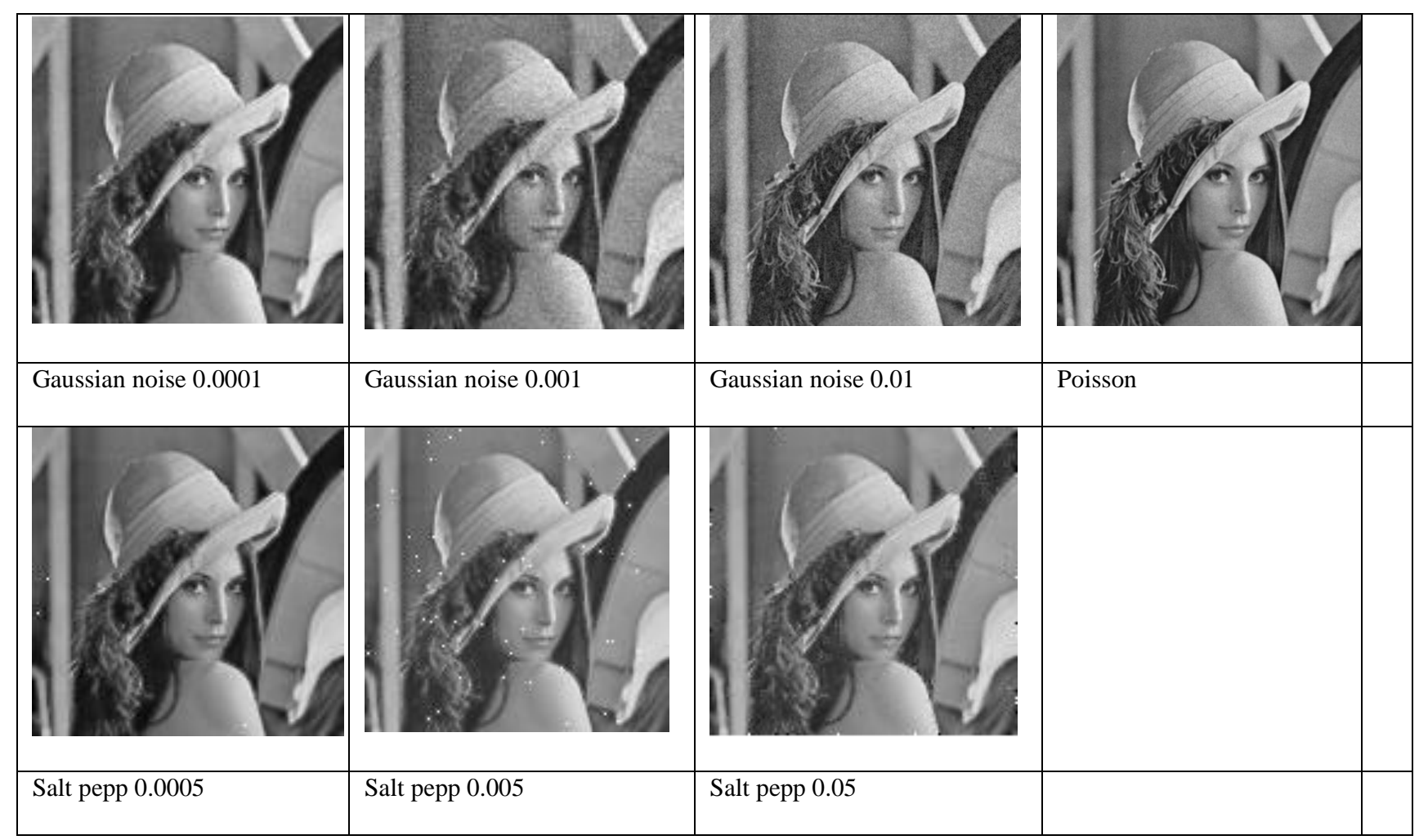




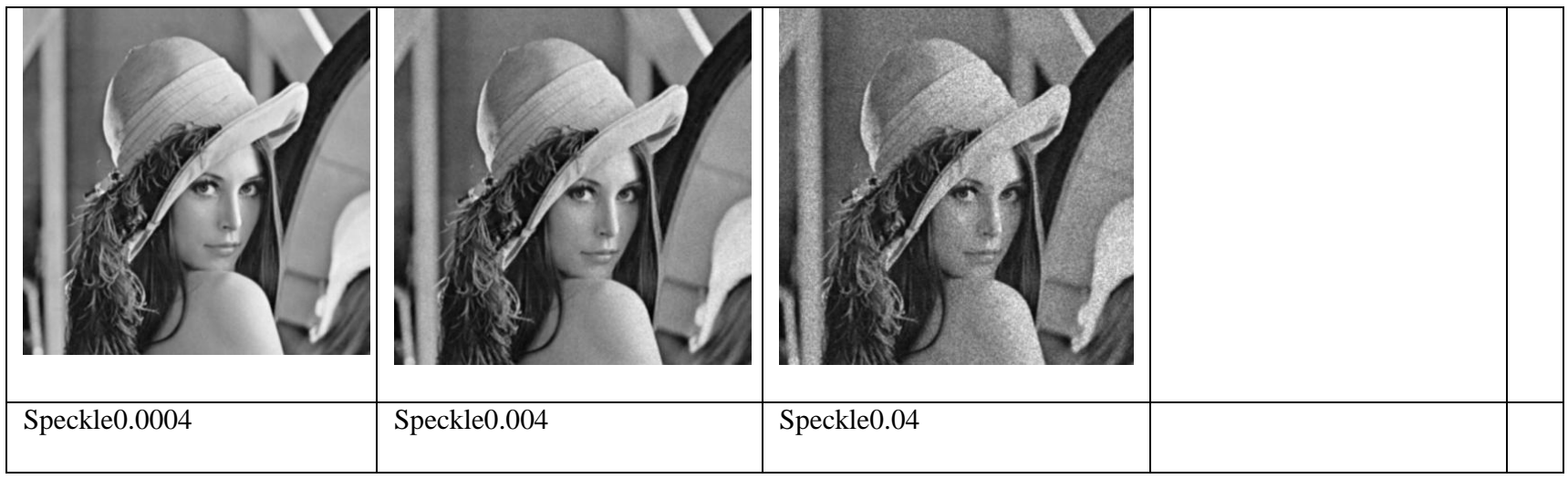

Fig 8: Denoising using Vertical variation with different noise density

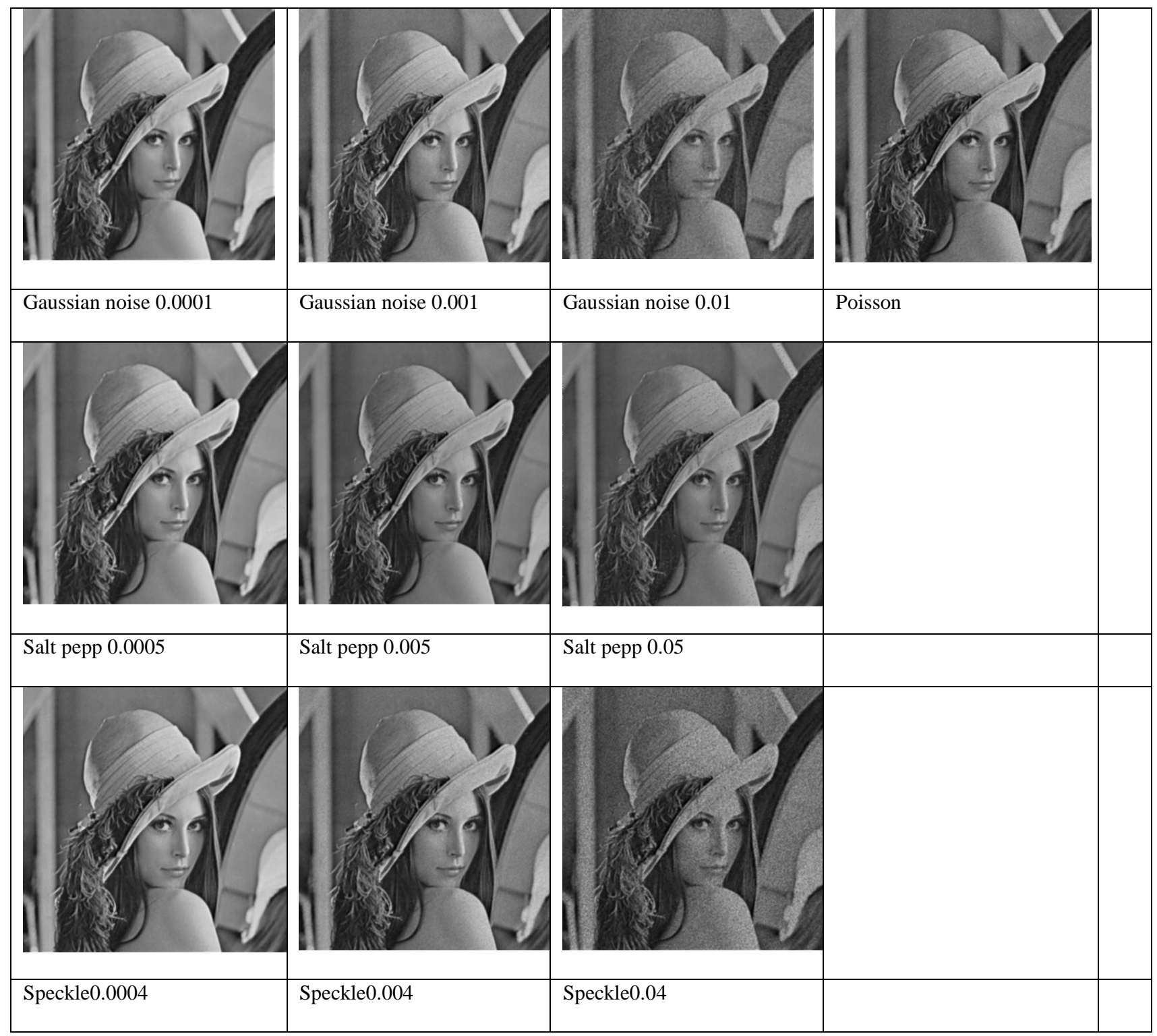

Fig 9: Denoising using Cluster variation with different noise density 